\title{
OC-041 HOXB CLUSTER GENES ARE NOVEL POTENTIAL REGULATORS OF BARRETT'S OESOPHAGUS DEVELOPMENT
}

doi:10.1136/gut.2011.239301.41

M Di Pietro, ${ }^{*}$ R C Fitzgerald Hutchison-MRC Research Centre, Cambridge, UK

Introduction Barrett's oesophagus $(\mathrm{BO})$ is a preneoplastic condition to oesophageal adenocarcinoma (OAC) where the squamous epithelium is replaced by a columnar epithelium as a consequence of chronic gastro-oesophageal reflux disease. The molecular events driving this metaplastic change are still poorly understood. Analysis of an in-house microarray screening showed activation in $\mathrm{BO}$ of three $\mathrm{HOXB}$ cluster genes (HOXB5, HOXB6 and HOXB7). Thus, we set out to validate whether these genes are causally linked to the development of $\mathrm{BO}$

Methods To externally validate microarray data, RNA was extracted from endoscopic biopsies of colon ( $n=14)$, duodenum $(n=13)$, gastric body $(n=11)$, normal oesophagus $(n=14)$, $\mathrm{BO}$ without dysplasia ( $\mathrm{n}=13$ ), $\mathrm{BO}$ with low grade dysplasia $(n=9)$ and high grade dysplasia $(n=6)$, as well as surgical specimens of OAC $(n=12)$. Expression of the mid cluster HOXB genes (HOXB5, HOXB6 and HOXB7), as well as neighbouring genes (HOXB4 and HOXB8), were assessed by qPCR. A normal oesophageal cell line (NES) was transiently transfected with vectors coding for HOXB5, HOXB6 and HOXB7 and the intestinal markers villin, cytokeratin 20 (KRT20), sucrase isomaltase (SI) and MUC2 were assessed by qPCR. Chromatin 
was isolated from surgical specimens of normal oesophagus ( $\mathrm{n}$ $=5)$ and $\mathrm{OAC}(\mathrm{n}=5)$ and immunoprecipitated with antibodies against methylated histone $\mathrm{H} 3$ at Lysine 27 (H3K27me3) and Lysine 4 (H3K4me3) and acetylated histone $\mathrm{H} 3$ (AcH3).

Results HOXB5, HOXB6 and HOXB7 genes were consistently up-regulated in all pathological stages of Barrett's disease, including OAC, as compared to normal oesophagus ( $p<0.0001$ for all three genes). HOXB4 and HOXB8 did not show any transcriptional activation in $\mathrm{BO}$ and $\mathrm{OAC}$. When compared with other GI segments, expression levels of these five HOXB genes in $\mathrm{BO}$ resembled that of the colon rather than other columnar epithelia (stomach and duodenum). Over-expression of mid cluster HOXB genes in NES cells was associated with activation of villin, KRT2 0 and MUC2 but not SI. In particular HOXB5 induced villin and MUC2 (2- and 4-fold, respectively); HOXB6 activated villin, KRT20 and MUC2 (4-, 3- and 4-fold, respectively) and HOXB7 induced KRT20 and MUC2 (14-fold for both). Chromatin immunoprecipitation showed significant enrichment of the active mark AcH3 and significant loss of the repressive mark $\mathrm{H} 3 \mathrm{~K} 27 \mathrm{me} 3$ in OAC compared to normal oesophagus for HOXB5, HOXB6 and HOXB7, but not HOXB4 and HOXB8. No change was detected for the active mark H3K4me3.

Conclusion Mid cluster HOXB genes are novel potential mediators of the preneoplastic intestinal phenotype in the oesophagus and they are regulated through an epigenetic mechanism acting on the histone code. The HOXB gene signature of oesophageal intestinal metaplasia is similar to that of large bowel, rather than small bowel and gastric epithelia.

Competing interests None. 